\title{
Fabrication of 3D-printed hygromorphs based on different cellulosic fillers
}

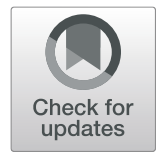

\author{
Matthias Langhansl ${ }^{1}$, Jörg Dörrstein ${ }^{1}$, Peter Hornberger ${ }^{2}$ and Cordt Zollfrank ${ }^{1^{*}}$ (D)
}

\begin{abstract}
The aim of this work is to characterize the moisture-dependent actuation behavior of bioinspired and additively manufactured hygromorphs based by following deductive and inductive design approaches. Fused Filament Fabrication (FFF) is employed to print bilayered structures consisting of swellable active layers and rigid passive layers. The active layer is composed of a polylactic acid (PLA) matrix filled with different hygroscopic cellulosic materials (native and modified) up to a filler content of $50 \mathrm{~m} \%$. Acrylonitrile Butadiene Styrene (ABS) is used for the passive layer. The FFF process allows the generation of desired differential swelling properties in the composites upon moisture absorption. The moisture dependent actuation strain of the printed bilayers was determined by video analyses. Some influencing geometrical factors which contribute to the actuation were deduced from $x$-ray diffraction (XRD) and micro computed tomography $(\mu \mathrm{CT})$. The investigation of the mean cellulose microfibril orientation on the surface of the active layer suggested a preferential orientation with respect to printing direction. Furthermore, a gradient of cellulosic material within a single printed layer was observed, which indicates fiber sedimentation. Comparison with the thermomechanical model derived from Timoshenko (1925) shows that the computational prediction of the moisture dependent actuation is considerably accurate for most selected cellulosic materials and filler contents.
\end{abstract}

Keywords: 3D printing, Actuation, Bilayer, Anisotropic swelling, Cellulose, Microcrystalline cellulose

\section{Introduction}

In nature plants have developed numerous kinds of movements to catch prey, to disperse seeds or to protect themself [1]. Many of these motions occur without an external source of energy. They are only driven by environmental humidity gradients [2]. The materials and design approaches inspired by these phenomena are often of non-renewable origin [3-5] while several approaches describe the use of wood $[6,7]$ or cellulose-based materials such as cottonid, which is even more hygroscopic then natural cellulose [8-10]. Hygromorphous structural materials respond to the relative humidity $(\Phi)$ of the environment by changing their dimensions, and thus their shape by swelling through the uptake of water. Pine cones are among the best known and well-described

\footnotetext{
* Correspondence: cordt.zollfrank@tum.de

${ }^{1}$ TUM Campus Straubing for Biotechnology and Sustainability, Biogenic

Polymers, D-94315 Straubing, Germany

Full list of author information is available at the end of the article
}

natural hygromorphs. The opening and closing of the ovuliferous scales is a result of the bilayered structure [11-13]. The different dimensional changes are caused by the almost perpendicular arrangement of cellulose fibrils in the adaxial (inner) and abaxial (outer) tissue. The humidity driven actuation of the scales can be described similar to thermally actuated bimetallic strips, where the two bound layers mechanically bend (mechanical energy) in response to differential expansion of the layers as a function of the temperature change (thermal energy) [14].. The classical modeling of the actuation of such bimetals according to Timoshenko 1925 [15] can be modified by replacing the actuation mechanism from temperature to humidity $[14,16]$. Herein the hygroexpansive strain is defined as the ratio between the changes in length to initial length as a function of moisture content [17]. By carefully characterizing geometry, mechanics, fluid transport, and evaporative flux of a porous medium the potential of natural and artificial 
hygromorphs can be estimated by employing hygromechanic models [14]. For the modeling of the actuation as a result of the sorption of moisture and dimensional changes a theoretical equation for thermo-bimetals was adjusted in the way that it is applicable for hygrosensitive actuators as reported earlier [14]. Following the assumption of Reyssat et al. the thickness of the active layer $h_{a}$ and the passive layer $h_{p}$, their moduli $E_{a}$ and $E_{p}$, and their hygrometric coefficients of linear expansion in their unrestrained state $\alpha_{\mathrm{a}}$ and $\alpha_{\mathrm{p}}$ are independent of relative humidity $\Phi$. Upon change in relative humidity $\Delta \Phi$ the isotropic expansion is given by $\alpha \Delta \Phi$. The curvature change of the bilayer $\Delta \kappa$ to calculate the theoretical displacement of moisture activated bilayers according to [14] is given by:

$$
\Delta \kappa=\frac{\alpha \Delta \phi \mathrm{f}(m, n)}{h}
$$

where

$$
f(m, n)=\frac{6(1+\mathrm{m})^{2}}{3(1+m)^{2}+(1+m n)\left(m^{2}+\frac{1}{m n}\right)}
$$

and

$$
m=\mathrm{h}_{\mathrm{p}} / \mathrm{h}_{\mathrm{a}}, n=\mathrm{E}_{\mathrm{p}} / \mathrm{E}_{\mathrm{a}}
$$

Provided that $\alpha=\alpha_{\mathrm{a}}-\alpha_{\mathrm{p}} \neq 0$ and $\mathrm{RH}_{\mathrm{t}=0} \neq 100 \%$ the differential expansion of two interconnected layers leads to a bending at the tip of the bilayer.

Recently 3D printing of wood and wood constituents like cellulose and lignin has attracted much attention due to their benign properties regarding low-cost and natural origin [18-20]. Moreover, the fabrication of programmable materials by exploiting anisotropic swelling which is an inherent material property of underivatized cellulose allows the generation of 3D-printed hygromorphs. Such materials may find applications in smart textiles, autonomous robotics, biomedical devices, drug delivery and tissue engineering $[21,22]$ and architecture [1]. However, further investigation is necessary to gain a better understanding of the behavior of the self-transforming composites. This consists in a detailed insight into the structure-transformation relationship which would allow the tailoring of hygromorphs preferably by adjusting the processing conditions. Additionally, the custom filament production allows for further experimentation on material composition as the use of different natural or modified cellulosic materials such as vulcanized cellulose and microcrystalline cellulose would impart different humidity-dependent dimensional changes [7].

\section{Material and methods Materials}

Polylactic acid (PLA, Ingeo 3251, NatureWorks LLC) and vinylacetate-vinylversatate-ethylene (Vac-VV-E, Vinnex LL 2505, Wacker Chemie AG) were used as received. The acrylonitrile-butadiene-styrene (ABS) filament (ABS black, Material 4 Print $\mathrm{GmbH}$ ) has a mean diameter of $3 \mathrm{~mm}$. As determined by microscopic image analysis microcrystalline cellulose (MCC, Alfa Aesar GmbH \& Co. KG) exhibit an average particle size of $27 \mu \mathrm{m}$, whereas Arbocel type F $140 \mathrm{~K}$ (AC, Rettenmaier \& Söhne $\mathrm{GmbH} \&$ Co. KG) have an average fiber length of $62 \mu \mathrm{m}$ and were used as received. SEM Images of the cellulosic fillers were taken on an Zeiss DSM 940 $A$ at $15 \mathrm{kV}$. The samples were gold sputter coated beforehand.

\section{Filament production}

The filament production was carried out using a corotating twin-screw microcompounder (HAAKE MiniLab II, Thermo Scientific). The microcompounder used for the fabrication of filaments was equipped with a custom-made nozzle with a diameter of $1.5 \mathrm{~mm}$ to compensate for the die swelling at the nozzle outlet, which is caused by entropic effects. A constant extrusion rate was maintained by setting the screw speed to $50 \mathrm{rpm}$ and the uptake with a bobbin facilitated the compensation of pulsation. Filaments with a diameter of $3 \mathrm{~mm} \pm 0.1 \mathrm{~mm}$ were produced, which was a prerequisite for reliable filament feeding throughout the printing process. The average residence time of the fiber-matrix mixture in the extruder was about $30 \mathrm{~s}$. Commercial black ABS was used as a passive layer.

\section{Fused filament fabrication}

Prior to the fabrication of the bilayers a digital model was created by using AutoCAD 2016 (Autodesk GmbH, Germany), Fig. 1 a. The model consisted of a passive layer of two perpendicularly oriented extrudate arrays of ABS, and of an active layer of PLA-cellulose compound. The bilayer model had the dimensions of $50 \mathrm{~mm} \times 50$ $\mathrm{mm} \times 0.75 \mathrm{~mm}$, with an overall thickness of the passive layer and the active layer of $0.5 \mathrm{~mm}$ and $0.25 \mathrm{~mm}$, respectively. For the exact determination of the actuation the protrusion of the passive layer was removed. Models created in AutoCAD 2016 further processed with the AXON2 program (3D systems Inc.). A 3D-printer operating according to the FFF principle (3D Touch, 3D systems Inc.) was used to print the bilayers. The diameter of the nozzle opening was $0.25 \mathrm{~mm}$. ABS and the active layer containing the PLA-cellulose composite were successively formed at $260^{\circ} \mathrm{C}$ and $210 \mathrm{C}$, respectively. Each bilayer formulation was printed and characterized in duplicate. 


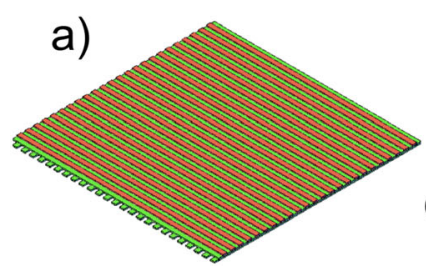

b)

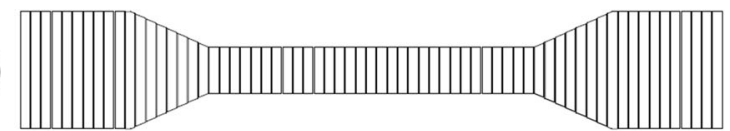

c)

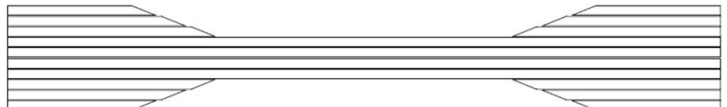

Fig. 1 a CAD-model of the PLA-based hygromorph consisting of mutually perpendicular passive layers (green) and an active layer (red). The length and width is $5 \mathrm{~cm}$. $\mathbf{b}$ and $\mathbf{c}$ Schematic illustration of the axial and radial tensile bars with the corresponing print paths for the determination of the elastic moduli

\section{Theoretical model}

According to eqs. (1) and (2), the determination of the elastic moduli of the individual layers Ea and Ep and the hygrometric coefficients of linear expansion $\alpha \mathrm{a}$ and $\alpha \mathrm{p}$ is needed. The elastic moduli of the individual layers were determined according to DIN EN 527 on six printed tensile bars (Fig. 1b) using a universal testing machine (smarTens, Karg Industrietechnik, Krailling, Germany). The results are given as the averages of six identically fabricated and tested specimens. The hygrometric coefficients of linear expansion were determined after waterimmersion of printed specimens $(100 \mathrm{~mm} \times 2 \mathrm{~mm} \times 0.5$ $\mathrm{mm})$ at room temperature for $1,2,3,20 \mathrm{~h}$ and measuring the dimensional change using a digital caliper with an accuracy of $0.02 \mathrm{~mm}$.

\section{Determination of the actuation}

The actuation (i.e. bending of the bilayer upon change in humidity) was followed by placing printed bilayers into a home-made climate chamber and subsequent video analysis. The humidity was established by placing dishes filled with water or different saturated salt solutions into the chamber. Humidity and temperature were measured constantly by a hygrometer 6100 (Electronic Temperature Instruments Ltd., Easting Close, UK). A tripod-mounted camera (Canon EOS 550D, Canon Germany GmbH, Krefeld, Germany) was connected to a PC. Images were taken at time intervals of $120 \mathrm{~s}$ with a total experimental time of $7 \mathrm{~h}$ (3.5 $\mathrm{h}$ for deflection and $3.5 \mathrm{~h}$ for provision). Each sample was determined in duplicate, and the results are given as averages. The camera operated with following settings:

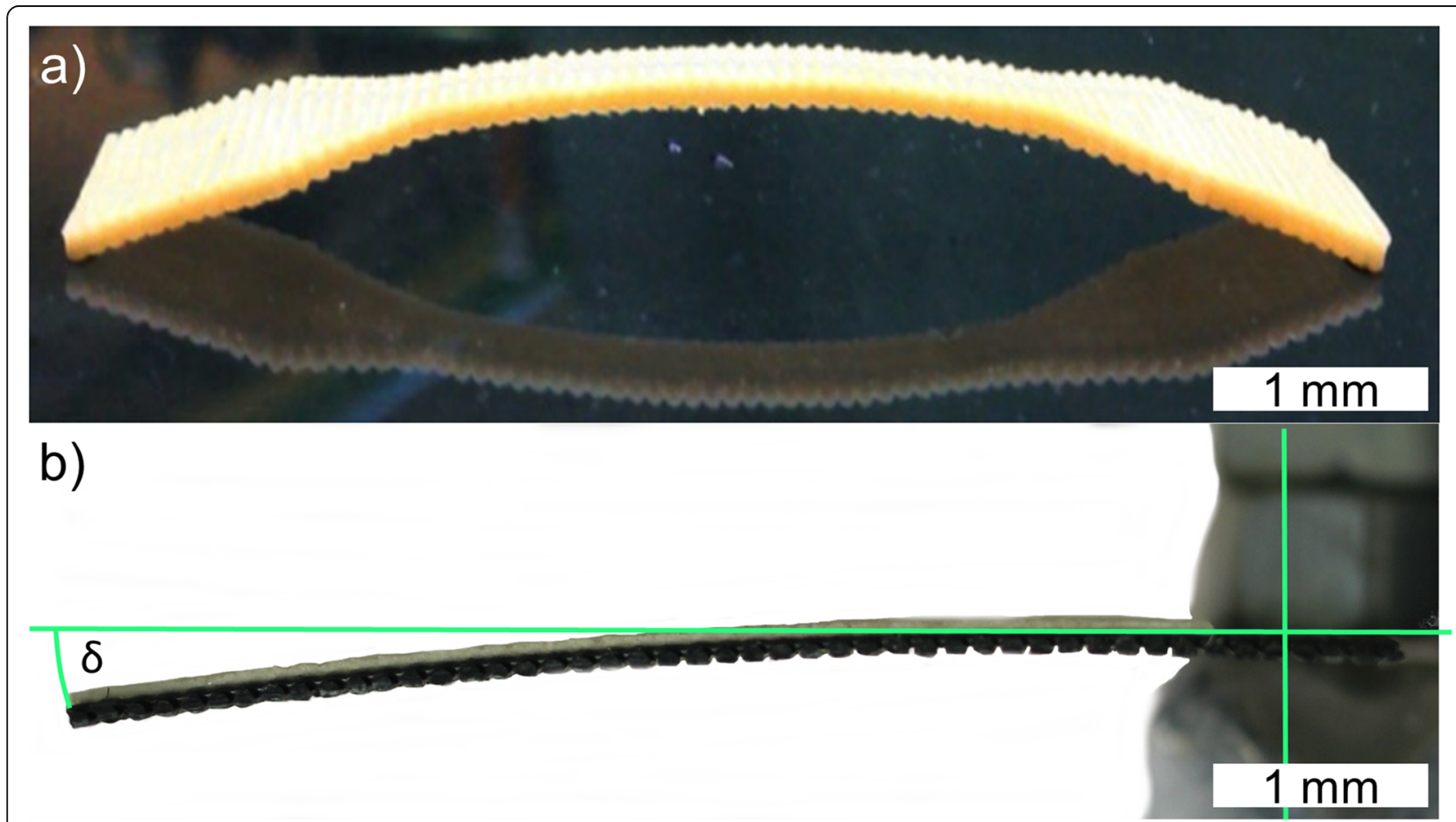

Fig. 2 a Illustration of an axial AC25\% hygromorph after water-immersion for $2 \mathrm{~min}$. $\mathbf{b}$ Example for the evaluation of the actuation of a 3Dprinted after storage in a climate chamber at $\mathrm{RH}=75 \%$. The green horizontal line represents the position of the bilayer prior to the change in humidity at $\mathrm{RH}=30 \%$ 
shutter speed $(\mathrm{Tv}) 1 / 25$, aperture $(\mathrm{Av}) 12$, sensitivity of the image sensor ISO 400. Subsequently, the resulting 210 pictures were converted to an avi video file with 30 frames per second. Finally, the videos were evaluated using the video analysis software Tracker (Douglas Brown, www.opensourcephysics.org), Fig. 2b.

\section{Apparent cellulose orientation Cellulose orientation close to the surface}

A Rigaku MniFlex 600 (Rigaku Corporation, Tokyo, Japan) X-ray diffractometer was used for the measurement. The sample was turned in steps of $10^{\circ}$ after each measurement in order to determine a possible orientation of the fibres. An irradiation angle $2 \theta$ of $5^{\circ}$ to $40^{\circ}$ was investigated. The step size was $0.1^{\circ}$ at a speed of $10^{\circ}$ per min at a voltage of $40 \mathrm{kV}$ and a current of $15 \mathrm{~mA}$. The intensity distribution is measured by obtaining the intensity at a fixed Bragg angle or $2 \theta$ as a function of the angle relative to the fibre axis direction (azimuthal angle). The used method of fibre alignment is the full width of the $(0,0,2)$ cellulosic plane azimuthal diffraction measured at one half of the maximium intensity. This measurement is usually designated as $\mathrm{Z}$ and given in degree. The measure represents the spread of the majority of the graphene planes. This value is the full spread of misalignment and should be thougt of as a cone angle because the alignment is in three dimensions. The value should be halved if one wishes to think of how far from the fibre axis the plans are misaligned [22, 23]. Thus, this method can give informations on the fiber orientation on the surface (limited by f.e. the excitation depth of the incident radiation) of the sample.

\section{Cross-sectional cellulose orientation}

In order to investigate the orientation of individual fibers throughout the entire active layer, the samples were subsequently analyzed by micro computed tomography $(\mu-\mathrm{CT})$. The $\mu-\mathrm{CT}$ measurement was carried out at the Fraunhofer Institute for Integrated Circuits IIS (Application Center CT in measurement technology (CTMT), Deggendorf). The scans were performed with a TomoScope HV 500 (Werth Messtechnik GmbH, Gießen Germany) tomograph. The parameters used for the measurements were: current $80 \mathrm{~A}$, voltage $180 \mathrm{kV}, 1600$ steps for a $360^{\circ}$ rotation and a total measurement time of $33 \mathrm{~min}$. The resolution of the images was dependent on the measurement and ranged from 8 to $12.5 \mu \mathrm{m}$. Scans were conducted on AC bilayer specimens with fiber contents of $25 \%$ and $50 \%$ to investigate a possible re-orientation of the fibers by the printing process and a $10 \mathrm{~cm}$ long piece of filament with $50 \%$ Arbocel to examine the distribution of the fibers in the filament over the cross section prior to the printing process. Furthermore, a cylindrical shaped printed specimen was investigated, to compare the results with XRD measurements.

\section{Results and discussion \\ Bilayer fabrication}

Preliminary tests using pure and fiber filled PLA showed that the brittleness of the filaments prevented proper feeding. Thus the impact strength of the composites was modified by the addition of $10 \mathrm{~m} \%$ of VAc-VV-E. Furthermore, the low glass transition temperature contributes to the processing at temperatures, preferably below the degradation of the cellulosic material starts. A $\mu$-CTscan of the extruded filament showed a homogeneous distribution of the cellulosic filler throughout its crosssections, Fig. 3. Cavitiy formation could not be observed within the cellulose filled filament. Figure $4 \mathrm{c}$ ) shows a cross section of a bilayer with $20 \%$ MCC filler. The connection between the active and the passivce layer is very good. The transition can only be recognized by the
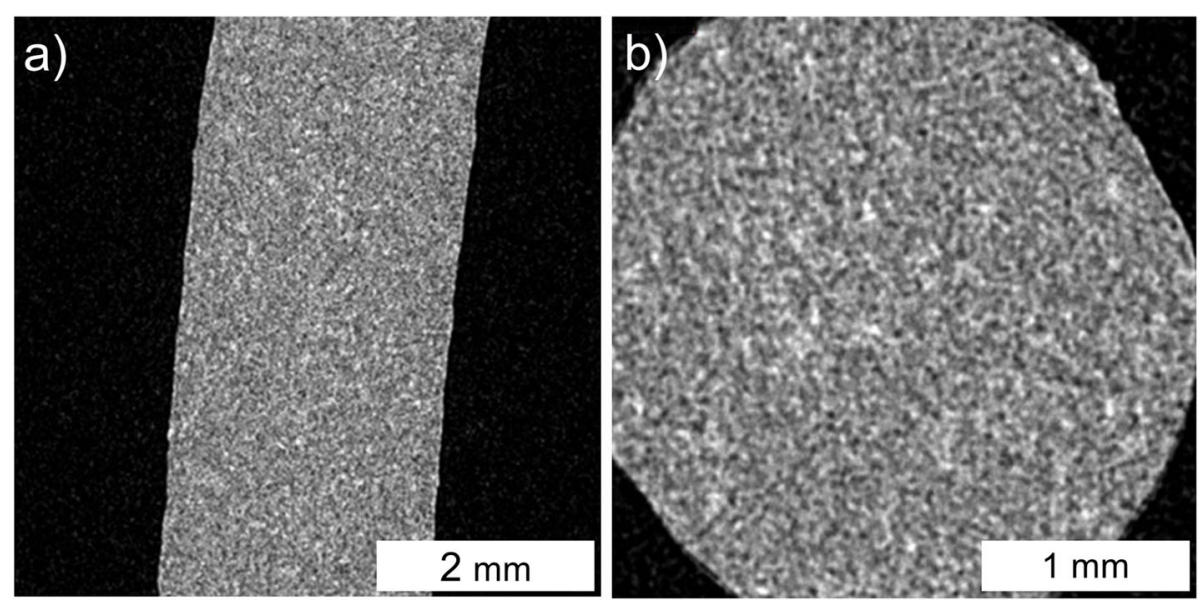

Fig. $3 \mu$-CT scan of AC50\% filament prior to printing: General overview of the filament form sample a and section through X-Y plane $\mathbf{b}$ Y-Z plane 

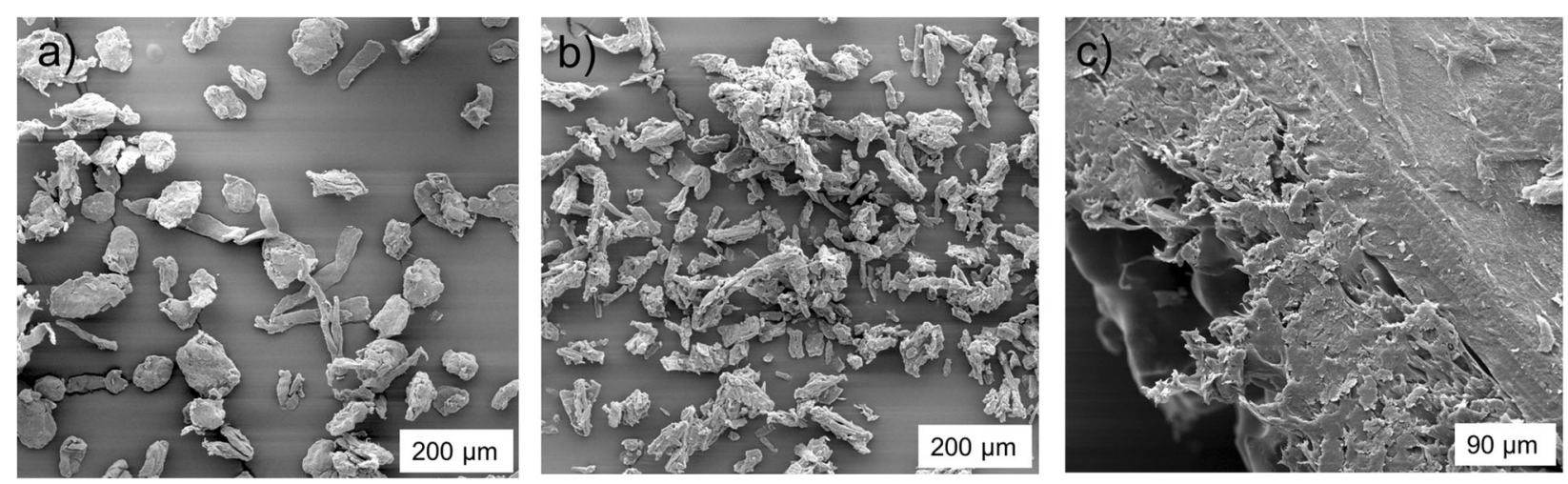

Fig. 4 SEM images of AC (a) and MCC (b) fibres. c shows the cross section of a $20 \%$ MCC bilayer

rougher fracture edge of the filled material as opposed to the sooth ABS.

\section{Actuation}

Figure 5 shows an example of the time-dependent angular change of printed bilayers with different amounts of fibers when exposed to a relative humidity of $75 \%$. All investigated actuation profiles showed a strong deflection directly after closing the climate chamber with $80 \%$. The highest deflection rate of $5 \% \mathrm{~min}$ observed in the first $60 \mathrm{~min}$. Subsequently, the angular velocity gradually decreased until the end of the measurement after 210 min. The resetting upon removal of the water dishes from the climate chamber was characterized by a higher absolute angular velocity of about $10 \%$ min. A relationship between fiber content and total deflection was found with the highest angular deflections of $6.5^{\circ}$ was observed at a fiber content of $50 \%\left(\mathrm{MCC}_{50 \%}\right)$, followed by $5.1^{\circ}\left(\mathrm{MCC}_{30 \%}\right)$, and $4.5^{\circ}\left(\mathrm{MCC}_{20 \%}\right)$. While the bilyers with $\mathrm{AC}$ fibres showed a maximum deflection of $3.0^{\circ}\left(\mathrm{AC}_{25 \%}\right)$ and $2.8\left(\mathrm{AC}_{50 \%}\right)$. The deflection of the matrix only sample was with $0.3^{\circ}$ very low. Therefore it can be assumed that the cellulosic fillers only cause the actuation. A complete return to the initial dimension of the samples was not reached by any of the samples under investigation within $300 \mathrm{~min}$. Only the matrix and the $\mathrm{AC}_{50 \%}$ samples reach almost the initial state. However, if the bilayer was stored at room temperature and a relative humidity of $30 \%$ a complete return was determined after approximately $10 \mathrm{~h}$. The MCC bilayers

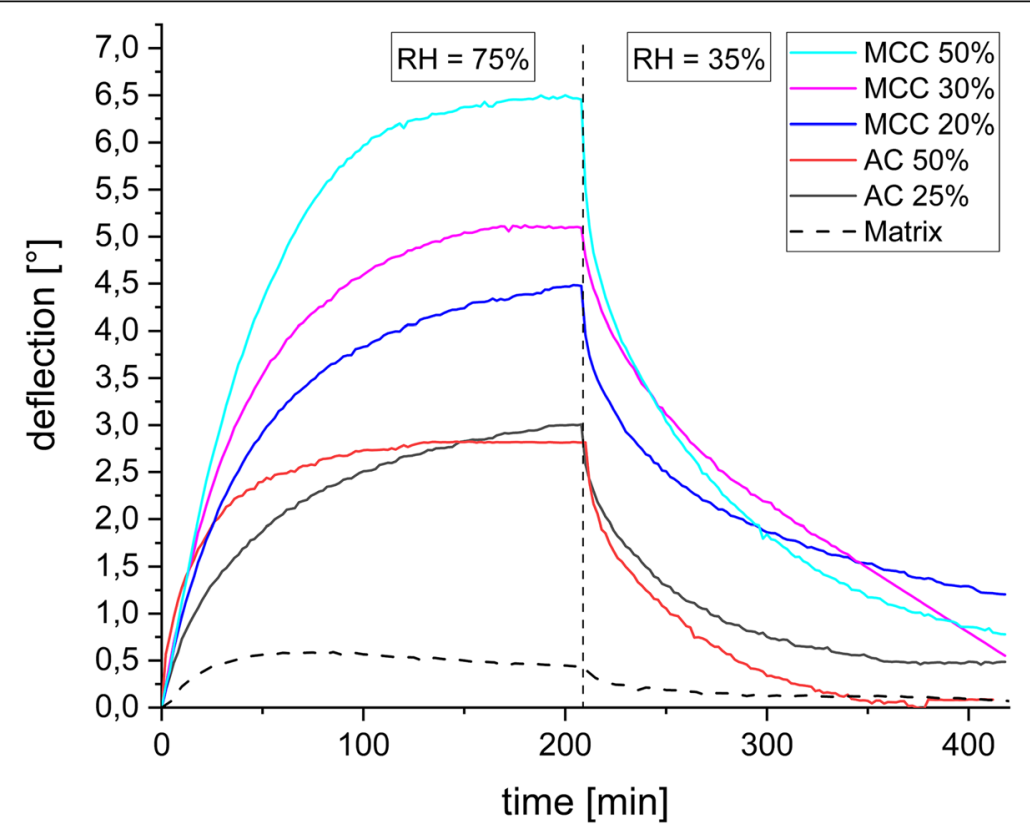

Fig. 5 Plot of the tip deflection versus time of all printed bilayers and the matrix during exposure to a relative humidity of $75 \%$ for 210 min and subsequent drying in ambient air by removing the water dish out of the climate chamber. The initial position of the equilibrated bilayer at ambient air was taken as the reference with $\theta_{\text {ambient air }}=0$ 
Table 1 Comparison of the measured curvature change $\Delta k$ measured of hygromorphs determined in a climate chamber and the modeled curvature change $\Delta$ kcalculated by determination of elastic moduli (E) and hygrometric coefficients of linear expansion of the active layer $\left(a_{a}\right)$

\begin{tabular}{|c|c|c|c|c|c|}
\hline Sample & $25 \%$ AC & $50 \%$ AC & $20 \% \mathrm{MCC}$ & $30 \% \mathrm{MCC}$ & $50 \% \mathrm{MCC}$ \\
\hline$\Delta \mathrm{K}_{\text {measured }}\left({ }^{\circ}\right)$ & 3.1 & 2.6 & 4.5 & 5.1 & 6.5 \\
\hline$\Delta \mathrm{K}_{\text {modeled }}\left({ }^{\circ}\right)$ & 3.2 & 3.1 & 3.1 & 4.8 & 6.4 \\
\hline $\mathrm{E}(\mathrm{MPa})$ & $458 \pm 35.9$ & $407 \pm 35.3$ & $289 \pm 42.7$ & $247 \pm 22.6$ & $478 \pm 56.0$ \\
\hline aa $(-)$ & 0.011 & 0.012 & 0.006 & 0.010 & 0.01 \\
\hline
\end{tabular}

showed the highest angular deflection, both relative to the mass related fiber content and in absolute values as mentioned before with $6.5^{\circ}$ observed at a fiber content of $50 \%\left(\mathrm{MCC}_{50 \%}\right)$. By comparison of the two fillers (Fig. 4) it can be seen that the MCC fibers are smaller than AC, which may cause the stonger actuation potential. What leads to a bigger amount of fibres for almost the same mass-percentage. The SEM image also show the more spherical shape of the AC filler, while in contrast to the MCC are more fibrous. This could also explain the poorer actuation properties of the $\mathrm{AC}_{50 \%}$ sample. The high fibre content can clump together, which considerably impairs the swelling capacity. The water uptake neither depends on the amount of fibres nor on their geometrie, all samples reach about $90 \%$ of their maximum curvature after about $100 \mathrm{~min}$.

\section{Theoretical model}

To understand the behavior of the actuators an analytical mechanical model was employed [10], which relates the curvature $(\mathrm{k})$ of the bilayer actuator to the humidityinduced strain of the material by eq. (1). The results of the $\mathrm{k}$-calculations are shown in Table 1 . The model showed a good match between calculated and measured curvature changes. The differences were partly ascribed to errors in the course of determination of elastic moduli of the individual layers Ea and Ep and the hygrometric coefficients of linear expansion $\alpha \mathrm{a}$ and $\alpha \mathrm{p}$ and defects during the processing occurred. In the case of MCC $30 \%$ and MCC50\% good matches were observed between measured and calculated curvature changes. However, a poor match was observed for MCC20\% which might be attributed to the comparatively lower fiber content which in turn leads to a reduced diffusion of water and therefore swellability of the microscale fibres. Comparing the E-moduli of the samples it is noticeable that the Emodulus of the $\mathrm{AC}$ samples decrease with increasing fibre content, while it increases with more fibres by MCC. This is probably due to the bigger size of the AC fibres, which are more easily entangled and cannot contribute to an increase of the stiffness. An exception is the sample with $30 \%$ MCC fibres, but considering the standard deviation it can be assumed that the two samples do not differ substantial form each other. Only an increase of the fiber to $50 \%$ cause an increase of the Emodulus by approximately $200 \mathrm{MPa}$. This is probably achieved by the smaller geometry of the MCC fibres, which embed themselves better in the matrix and tend to tangle less.

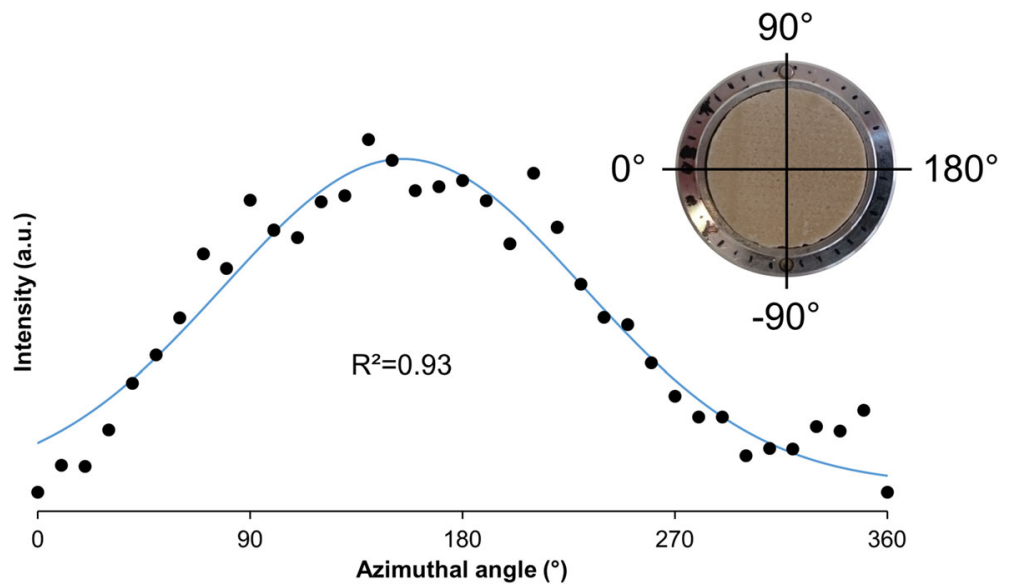

Fig. 6 Diffraction intensity (a.u.) versus azimuthal angle $\left({ }^{\circ}\right)$ of the AC50\% specimen and the Gauss-shaped fitting curve. The fiber alignment is calculated by the value of the angle (Z) for the width of the diffraction at one half the maximum intensity 
Table 2 Fiber orientation (Z/2) along the preferred axis of the AC25\% and AC50\% hygromorphs. A comparison of the upperside and underside of fabricated AC25\% specimens showed slight differences for Z/2

\begin{tabular}{llll}
\hline Sample & AC25\% Upperside & AC25\% Underside & $\begin{array}{l}\text { AC50\% } \\
\text { Upperside }\end{array}$ \\
\hline$Z\left(^{\circ}\right)$ & 192 & 178 & 180 \\
$Z / 2\left(^{\circ}\right)$ & 96 & 89 & 90 \\
R2 & 0.77 & 0.96 & 0.93 \\
\hline
\end{tabular}

\section{Fiber orientation}

To investigate the orientation of the cellulose fibrils close to the surface the diffraction intensity of the 002 peak (a.u.) versus azimuthal angle $\left(^{\circ}\right.$ ) of the $\mathrm{AC}_{50 \%}$ specimen is shown in Fig. 6.

A Gaussian-shaped fitting curve yields the orientation angle $\mathrm{Z} / 2$ and gives information on fiber alignment. The results from $x$-ray diffraction analyses are summarized in Table 2.

Interestingly, the results obtained from XRD suggest that the orientation of cellulose fibrils close to the surface is perpendicular to the direction of printing. However, further investigation is needed which may also gain more understanding into the nonrecurring peak at $0^{\circ}$ which we should assume for aligned discontinuous fiberreinforced composites [22].
To obtain additional information on the fiber orientation throughout the XRD-samples of $\mathrm{AC} 25 \%, \mathrm{AC}_{50 \%}$, $\mathrm{MCC}_{10 \%}$ and $\mathrm{MCC}_{20 \%}$ were studied by $\mu$-CT, shown in Fig. 7. The AC25\% showed a graduated filler distribution with successively lower filler contents towards the bottom of the printed part. A possible explanation is that during printing the layer-by-layer deposition of successive filled material leads to a reheating of the layers and consequently to a gradual sedimentation of the filler to bottom. This graduation was not observed in the AC50\% specimen neither in the $\mathrm{MCC}_{20 \%}$ sample. Here, a homogeneous distribution of the cellulosic filler is clearly observed with relatively uniform distribution within a plane. The same results for the $\mathrm{MCC}_{20 \%}$ sample. Only the ct-scan of the $\mathrm{MCC}_{! 0 \%}$ sample shows a lack of fibres between the single layers.

\section{Summary}

The aim of this work was to characterize the moisturedependent actuation behavior of bioinspired and additively manufactured hygromorphs. Printable filaments were produced using a microcompounder which contained different quantities of pretreated and untreated cellulose fibers. The brittleness of the PLA was adjusted by the addition of a terpolymer of vinyl acetate-ethylenevinyl versatate $(10 \mathrm{~m} \%)$, in order to improve the processability. FFF was used to successfully fabricate bilayered
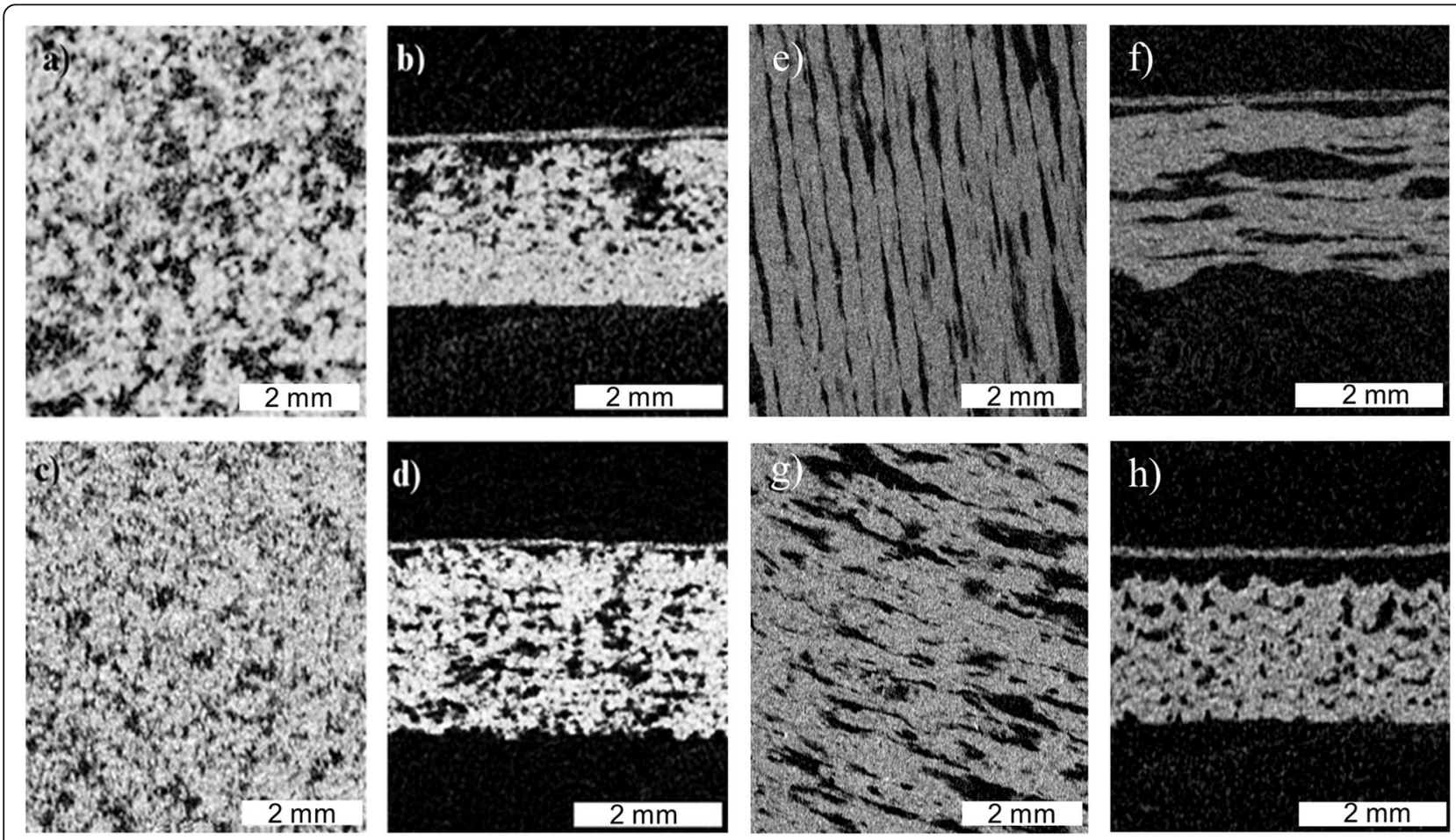

Fig. 7 Cross-sectional $\mu-C T$ images of 3D-printed XRD samples displaying the fibre dispersion within AC25\% (a, b),AC50\% (c, d), MCC10\% (e,f) and MCC20\% ( $\mathbf{g}, \mathbf{h})$ as sections through the X-Y plane $(\mathbf{a}, \mathbf{c}, \mathbf{e}, \mathbf{g})$ and $Y-Z$ plane $(\mathbf{b}, \mathbf{d}, \mathbf{f}, \mathbf{h})$ 
hygromorphous materials inspired by naturally occurring actuators. The analysis of these actuators suggest the presence of a specific preferential direction of the cellulose fibrils within the active layer, which might contribute to the actuation. Fiber sedimentation suggest to use mono-materials to create graded structures which in turn lead to actuation by omitting a rigid counterlayer. Finally, it could be shown that the actuation behavior of the printed hygromorphs can be estimated with high confidence by employing hygromechanic models.

\section{Acknowledgements}

Not applicable.

\section{Authors' contributions}

J. Dörrstein conceived the topic. P. Hornberger performed the CT-scans. M. Langhansl drafted the article, conducted the Experiments and analized the data. C. Zollfrank supervised the project. The authors read and approved the final manuscript.

\section{Funding}

The authors thank the German Research Foundation (Deutsche Forschungsgemeinschaft, DFG) for funding the research project "Biomechanical qualification of the structure-optimized functional material Cottonid as an adaptive element" (WA 1672/23-1; ZO 113/22-1) and "Mechanism-based development and validation of a structural health monitoring for climate adaptive architectural Cottonid elements" (WA 1672/23-2; ZO 113/22-2). Open Access funding enabled and organized by Projekt DEAL.

\section{Availability of data and materials}

The datasets used and/or analysed during the current study are available from the corresponding author on reasonable request.

\section{Competing interests}

The authors declare that they have no competing interests.

\section{Author details}

${ }^{1}$ TUM Campus Straubing for Biotechnology and Sustainability, Biogenic Polymers, D-94315 Straubing, Germany. ${ }^{2}$ Application Center for CT in Metrology, Fraunhofer Institute for Integrated Circuits, IIS, D-94469

Deggendorf, Germany.

Received: 8 September 2020 Accepted: 17 December 2020

Published online: 29 January 2021

\section{References}

1. S. Poppinga et al., Toward a New Generation of Smart Biomimetic Actuators for Architecture, vol 1703653no. Icd (2017), pp. 1-10

2. Y. Forterre, Slow, fast and furious: Understanding the physics of plant movements. J. Exp. Bot. 64(15), 4745-4760 (2013)

3. L. Zhang, S. Chizhik, Y. Wen, P. Naumov, Directed motility of Hygroresponsive biomimetic actuators. Adv. Funct. Mater. 26(7), 1040-1053 (2016)

4. B. Shin, J. Ha, M. Lee, K. Park, G. H. Park, T. H. Choi, K-J. Cho, H.-Y. Kim, Hygrobot: A self-locomotive ratcheted actuator powered by environmental humidity. Sci. Robot. 3, eaar2629 (2018)

5. S. Li, K.W.W. Wang, Plant-inspired adaptive structures and materials for morphing and actuation: A review related content kinematic amplification strategies in plants and engineering fluidic origami: A plant inspired adaptive structure with shape morphing and stiffness tuning. Bioinspir. Biomim 12, 11001 (2017)

6. A. Holstov, B. Bridgens, G. Farmer, Hygromorphic materials for sustainable responsive architecture. Constr. Build. Mater. 98, 570-582 (2015)

7. A. Papadopoulou, C. Guberan, N. Jhaveri, and D. Correa, "3D-Printed Wood: Programming Hygroscopic Material Transformations," vol. 2, 3, pp. 106,-116 2015
8. R. Scholz, M. Langhansl, C. Zollfrank, F. Walther, Experimental study on the actuation and fatigue behavior of the biopolymeric material Cottonid. Mater. Today Proc. 7, 476-483 (2019)

9. R. Scholz, J. Kristin, Direction-Dependent Mechanical Characterization of Cellulose- Based Composite Vulcanized Fiber, vol 58 (2016), pp. 813-817

10. R. Scholz, M. Langhansl, C. Zollfrank, F. Walther, L.M. Vergani, F.M. Curà, Humidity-Sensing Material Cottonid - Microstructural Tuning for Improved Actuation and Fatigue Performance, vol 7 (2020), pp. 1-10

11. W.N.I. Harlow, W. Cote, A. Day, The opening mechanism of pine cone scales. J. For., 538-540 (1964)

12. S. Poppinga et al., Hygroscopic motions of fossil conifer cones. Sci. Rep. 7no. December 2016, 5-8 (2017)

13. S. Poppinga et al., Compliant Mechanisms in Plants and Architecture (Springer, Cham, 2016), pp. 169-193

14. E. Reyssat, L. Mahadevan, Hygromorphs : From Pine Cones to Biomimetic Bilayers (2009)

15. S. Timoshenko, Analysis of bi-metal thermostats. J. Opt. Soc. Am. 11(3), 233 (1925)

16. I. Burgert, P. Fratzl. Actuation systems in plants as prototypes for bioinspired devices. Philos. Trans. A Math. Phys. Eng. Sci. 367(1893), 1541-1557 (2009)

17. M.M. Hamedi et al., Electrically activated paper actuators. Adv. Funct. Mater. 26(15), 2446-2453 (2016)

18. M. Kariz, M. Sernek, M.K. Kuzman, Use of wood powder and adhesive as a mixture for 3D printing. Eur. J. Wood Wood Prod. 74(1), 123-126 (2016)

19. D. Wood, C. Vailati, A. Menges, M. Rüggeberg, Hygroscopically actuated wood elements for weather responsive and self-forming building parts Facilitating upscaling and complex shape changes. Constr. Build. Mater. 165, 782-791 (2018)

20. D. Correa et al., 3D-printed Wood: Programming hygroscopic material transformations. 3D Print. Addit. Manuf. 2(3), 106-116 (2015)

21. T. Gladman, Precipitation hardening in metals. Mater. Sci. Technol. 15(1), 30-36 (1999)

22. R.J. Kuriger, M.K. Alam, D.P. Anderson, Strength prediction of partially aligned discontinuous fiber-reinforced composites. J. Mater. Res. 16(1), 226232 (2001)

23. J.J. Vilatela, L. Deng, I.A. Kinloch, R.J. Young, A.H. Windle, Structure of and stress transfer in fibres spun from carbon nanotubes produced by chemical vapour deposition. Carbon N. Y. 49(13), 4149-4158 (2011)

\section{Publisher's Note}

Springer Nature remains neutral with regard to jurisdictional claims in published maps and institutional affiliations.

\section{Submit your manuscript to a SpringerOpen ${ }^{\circ}$ journal and benefit from:}

- Convenient online submission

- Rigorous peer review

- Open access: articles freely available online

High visibility within the field

- Retaining the copyright to your article

Submit your next manuscript at $\boldsymbol{\triangleright}$ springeropen.com 